\title{
Polarization Patterns of Pi 2 Magnetic Pulsations and the Substorm Current Wedge
}

\author{
Mark Lester and W. Jeffrey Hughes \\ Department of Astronomy, Boston University
}

Howard J. Singer

Air Force Geophysics Laboratory

\begin{abstract}
The results of an analysis of 16 Pi 2 pulsations observed with an extended east-west chain of midlatitude, ground-based magnetometers are reported. The events were chosen such that the center of the substorm current system, defined by using the midlatitude bays associated with these pulsations, was within the longitudinal extent of the station chain. A current wedge model is also used to interpret the observed polarization pattern of the $\mathrm{Pi} 2$ pulsations. This pattern is used to locate the center of the Pi 2 current system. The centers of the Pi 2 and substorm current systems occur at the same meridian for only $\sim 65 \%$ of the events. This result suggests that the Pi 2 current system and the substorm current wedge are not always the same. The longitudinal extent of the $\mathrm{Pi} 2$ current system estimated from the Pi 2 polarization pattern averages $\sim 90^{\circ}$ or $\sim 6$ hours local time for the events in the study. The sense of ellipticity of the waves, anticlockwise looking down the field line in the northern hemisphere, agrees with previous results from these latitudes. Estimates of the wave-phase difference between stations show that, in general, the eastern station of a station pair leads the western one at all local times for both $H$ and $D$ components. When plotted in a substorm coordinate system based on the mid-latitude bay, the phase difference per degree of longitude shows a tendency to decrease in the eastern portion of the current wedge. This longitudinal pattern of phase difference is consistent with the eastern, downward, field-aligned currents being less localized than the western, upward, field-aligned currents.
\end{abstract}

\section{INTRODUCTION}

The appearance of $\mathrm{Pi} 2$ magnetic pulsations at the onset of substorms has been recognized for many years [Saito, 1961]. Recently, a consensus [Rostoker et al., 1980] included at least one $\mathrm{Pi} 2$ pulsation event as an integral part of a substorm, and the $\mathrm{Pi} 2$ signature has been used to time substorm onsets [Rostoker and Olson, 1979, and references therein]. Because of its association with substorm onset, the study of this class of pulsation is important for the understanding of substorm dynamics. In this paper we report observations of Pi 2 pulsations made with the AFGL (Air Force Geophysics Laboratory) east-west chain of mid-latitude magnetometers [Knecht et al., 1979] to investigate the relationship between the waves and the substorm current system [McPherron et al., 1973; Baumjohann et al., 1981].

The AFGL magnetometer chain is particularly well suited for the study of $\mathrm{Pi} 2$ pulsations for several reasons. The chain extends over 4 hours magnetic local time. This makes it useful for studying the three-dimensional current systems associated with Pi 2 pulsations. Also, since Pi 2 pulsations can often be observed over a large portion of the nightside on the ground [Saito et al., 1976; Sutcliffe, 1980; Singer et al., 1983] the chain can be used to study the longitudinal variations in the wave structure. Finally, the mid-latitude location of the stations is a region where the Pi 2 signal is relatively free from contamination caused by currents in the auroral ionosphere [Samson, 1982], and consequently the mid-latitude signal provides clearer results than the auroral zone signature [Samson and Harrold, 1983].

The three-dimensional substorm current wedge has been

Copyright 1983 by the American Geophysical Union.

Paper number 3A1061.

0148-0227/83/003A-1061\$05.00 described by McPherron et al. [1973], who locate the center of the wedge at the longitude where there is a change in sign of the east-west magnetic bay. If it is assumed that the $\mathrm{Pi} 2$ pulsation results from an oscillation of this current system [Bostrom, 1972], then the mid-latitude Pi 2 horizontal polarization pattern should exhibit a predictable longitudinal pattern. The predicted polarization pattern provides another technique for locating the center of the wedge [Knecht, 1981]. Comparing the two techniques, we find that the methods agree in only approximately two thirds of the cases. Little previous work has made use of the substorm current wedge to order the Pi 2 polarization [Knecht, 1981; Samson and Harrold, 1983], although in the past, several apparent local time variations have been reported [Bjornsson et al., 1971; Fukunishi, 1975; Baranskiy et al., 1980; Stuart and Baranskiy, 1982].

The phase differences between stations for $\mathrm{Pi} 2$ pulsations are examined. Although there have been some differences in the results of previous studies [Herron, 1966; Mier-Jedrzejowicz and Southwood, 1979; Baranskiy et al., 1980], the predominant observation is that eastern stations lead in phase. This result has been interpreted as western phase propagation on the entire nightside. Since no physical interpretation consistent with substorm models has been developed, we concluded that a further study of phase variations was warranted. Our results confirm that eastern stations lead in phase, but that the phase variation is not uniform across the substorm current wedge.

The following section describes the magnetometer array and data set used. We then describe the model of the substorm current wedge and the associated, predicted Pi 2 polarization pattern. Examples of $\mathrm{Pi} 2$ pulsations, their polarization pattern, and the related bay structure are then given. The polarization pattern is described for 16 events where it is possible to locate the center of the substorm 


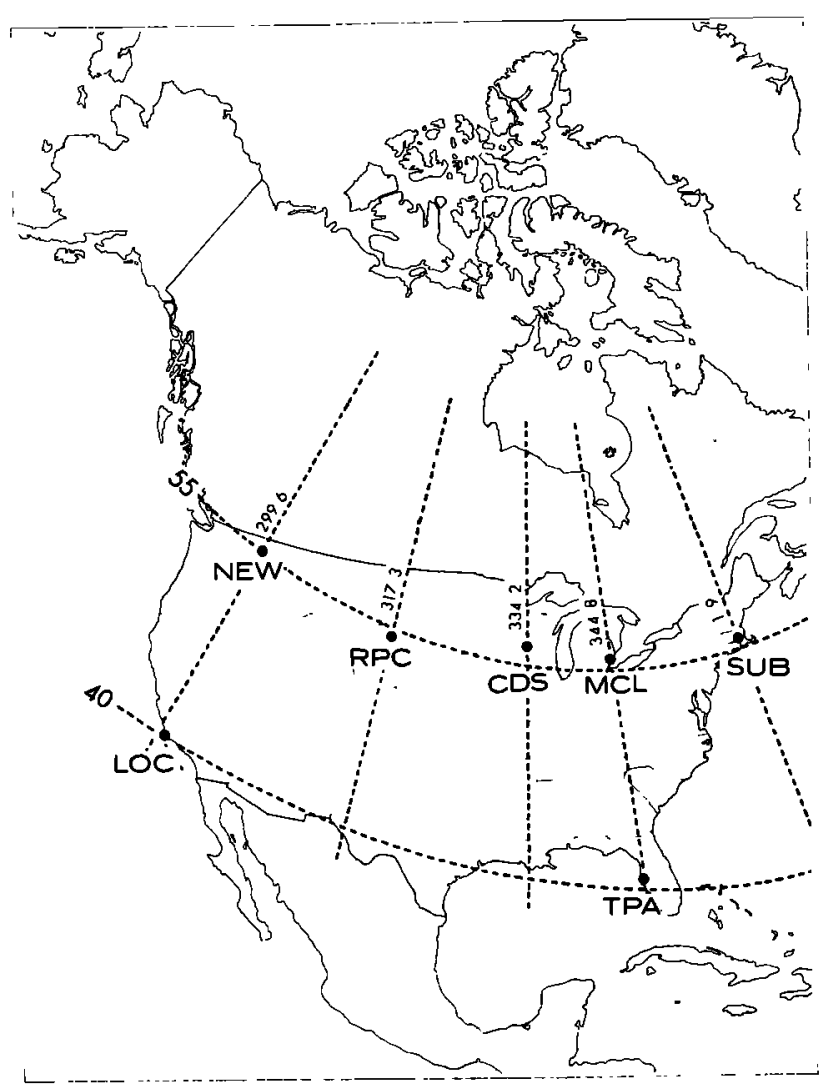

Fig. 1. Map showing the locations of the AFGL network stations. The five northern stations, extending over 4 hours local time, are at $-55^{\circ}$ corrected geomagnetic latitude; the two southern stations, which are not used in this study, are at $\sim 40^{\circ}$.

current system between the two extreme meridians of the network. Finally our results are discussed in the context of previous studies and the three-dimensional current system model.

\section{The Data Set}

The source of data for the study is the AFGL magnetometer network [Knecht et al., 1979]. The geographic and corrected geomagnetic coordinates of the seven AFGL stations are given in Table 1 . Five of these stations are at $\sim 55^{\circ}$ corrected geomagnetic latitude, and the remaining two stations are at $\sim 40^{\circ}$ (Figure 1). The latitudes of the stations place them all on field lines which thread the plasmasphere, although it is possible that during extremely disturbed magnetic conditions the plasmapause may move far enough radially inward to place the field lines of the northern stations in the plasma trough. Data from the two southern stations are not used in this study. The total longitudinal separation of the five northern stations is $62.3^{\circ}$ corrected geomagnetic longitude, which corresponds to slightly more than $\mathbf{4}$ hours magnetic local time. The longitudinal separations between adjacent stations at the same latitude are given in Table 1.

At each station a three-component flux gate magnetometer samples the field once a second with an amplitude resolution of $\sim 0.06 \mathrm{nT}$. The three components are $H(+v e$ geomagnetic north), $D$ ( $+v e$ geomagnetic east), and $Z$ ( $+v e$ vertically down). This study uses 5-s averages. The time interval from which the data were taken is March 14-19, 1978. During this interval, we identified more than $40 \mathrm{Pi} 2$ pulsations at one or more stations.

\section{The Substorm Current Wedge CoORdinate SySTEM}

In many previous investigations of $\mathrm{Pi} 2$ pulsations, local time has been used to order Pi 2 parameters such as the horizontal wave ellipticity [e.g., Fukunishi, 1975; Lester and Orr, 1981], the orientation of the major axis of the horizontal ellipse [e.g., Baranskiy et al., 1980; Stuart and Baranskiy, 1982], and the phase difference between stations [e.g., Herron, 1966; Mier-Jedrzejowicz and Southwood, 1979], with occasional differences between studies being reported. Since $\mathrm{Pi} 2$ pulsations are associated with substorms, it seems natural to use the position of the stations relative to some substorm feature to better organize the data. Features such as the auroral breakup region [Kuwashima and Saito, 1981] or the auroral electrojet [Rostoker and Samson, 1981; Samson and Rostoker, 1983] would be useful, but no method of reliably locating them with mid-latitude data is available. However, with an east-west chain of mid-latitude magnetometers, one can locate the position of the substorm current wedge by using a model described by McPherron et al. [1973]. The local time of isolated substorm onsets can vary substantially, and there is usually a westward motion during a sequence of substorm intensifications [Wiens and Rostoker, 1975; Knecht, 1981]. Consequently, organizing the $\mathrm{Pi} 2$ parameters by using the location of the wedge relative to a station has clear advantages over the use of local time.

The simple current system discussed by McPherron et al. [1973] and developed further by Clauer and McPherron $[1974 a]$ is used as the model and is shown in Figure 2. At the onset of the expansion phase of the substorm the crosstail current is diverted down the magnetic field lines. Current then flows in the ionosphere as the westward electrojet and returns to the tail along the magnetic field lines. The perturbation tail current can be represented by an equivalent eastward current, which completes the three-dimensional current wedge. Although this current system is an oversimplification several authors [e.g., McPherron et al., 1973;

TABLE 1. Name and Coordinates of AFGL Magnetometer Network Stations

\begin{tabular}{lcccccc}
\hline & \multicolumn{2}{c}{ Geographic } & & \multicolumn{2}{c}{ Corrected Geomagnetic } & \\
\cline { 2 - 3 } \multicolumn{1}{c}{ Station Name } & $\begin{array}{c}\text { East } \\
\text { Longitude }\end{array}$ & $\begin{array}{c}\text { North } \\
\text { Latitude }\end{array}$ & & $\begin{array}{c}\text { East } \\
\text { Longitude }\end{array}$ & $\begin{array}{c}\text { North } \\
\text { Latitude }\end{array}$ & $\begin{array}{c}\text { Station } \\
\text { Separation }\end{array}$ \\
\hline NEW, Newport, WA & $242.9^{\circ}$ & $48.3^{\circ}$ & & $299.6^{\circ}$ & $55.2^{\circ}$ & 17.7 \\
RPC, Rapid City, SD & 256.9 & 44.2 & & 317.3 & 54.1 & 16.9 \\
CDS, Camp Douglas, WI & 269.7 & 44.0 & & 334.2 & 56.3 & 10.6 \\
MCL, Mt. Clemens, MI & 277.1 & 42.6 & & 344.8 & 55.8 & 17.1 \\
SUB, Sudbury, MA & 288.7 & 42.2 & & 1.9 & 55.8 & \\
LOC, Lompoc, CA & 239.4 & 34.7 & & 300.6 & 40.2 & 44.3 \\
TPA, Tampa, FL & 277.5 & 27.8 & & 344.9 & 40.7 & \\
\hline
\end{tabular}



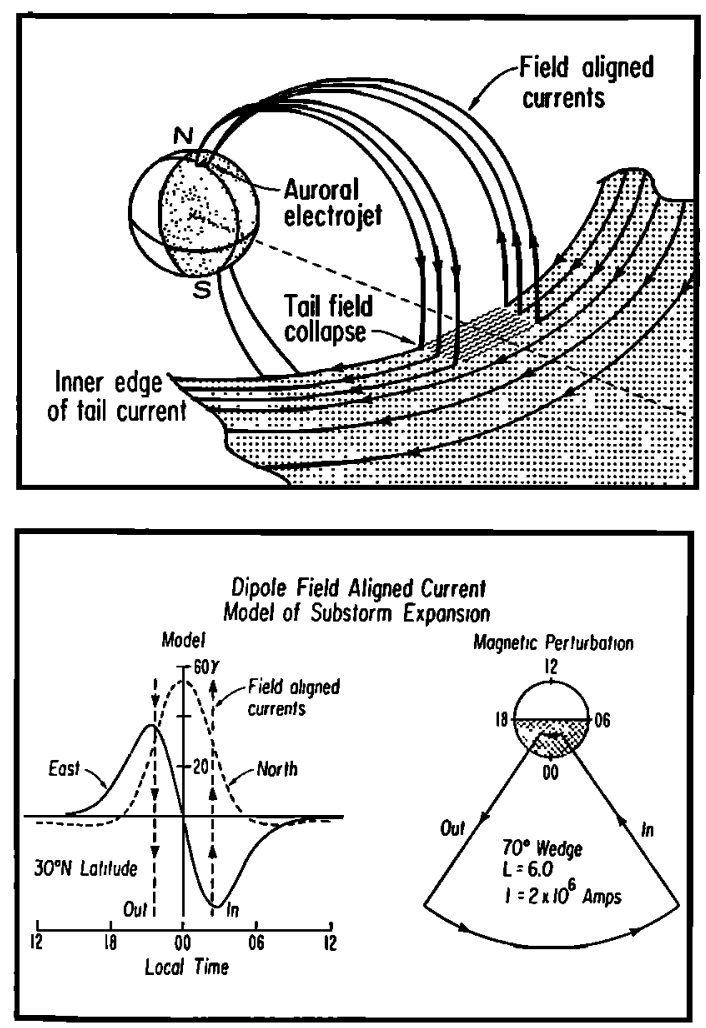

Fig. 2. The upper panel shows schematically the model of the substorm current wedge which is used to position the stations relative to the center of the substorm wedge. The bottom panel (right) shows an equivalent current system and model parameters. The bottom panel (left) summarizes the calculated sense and magnitude of the mid-latitude magnetic bay expected after substorm onset [from Clauer and McPherron, 1974a].

Clauer and McPherron, 1974a] have shown that it accurately describes mid-latitude bay variations. The mid-latitude signature of this wedge is a positive perturbation in the northsouth magnetic component symmetric about the central meridian of the current system and a perturbation in the eastwest magnetic component that is positive to the west and negative to the east of the central meridian.

Figure 3 shows schematically the field-aligned and ionospheric legs of the substorm current wedge and the magnetic variations caused by the two field-aligned currents. The field-aligned currents will, ideally, only affect the horizontal magnetic components at mid-latitudes. If the $\mathrm{Pi} 2$ pulsation is directly associated with the substorm current system, then it will have the predicted polarization pattern across the current wedge shown in Figure 3. This pattern can be tested by using events where the center of the current wedge, located by the bay current system, is between the two longitudinal extremes of the magnetometer chain.

In order to quantify the bay structure for each Pi 2 event we measured both the $H$ and $D$ values at the five northern stations at the start of the $\mathrm{Pi} 2$ pulsation and at a time when no more wave activity was observed. We defined the difference between the two values as $\Delta H$ and $\Delta D$. The time difference was typically between 10 and 15 minutes. The central meridian is where $\Delta D=0$. The $H$ perturbation provides a consistency check, i.e., $\Delta H$ should be positive and maximize near the location of $\Delta D=0$. Of the original 40 events, 32 had such a bay structure, and for 16 of these 32 the location of $\Delta D=0$ was between the NEW and SUB meridians.

The longitude of $\Delta D=0$ was calculated by assuming a linear change in $\Delta D$ between the two stations on either side of $\Delta D=0$. Each station was located in longitude, relative to the meridian where $\Delta D=0$, by calculating the longitude difference: $\Delta$ long. $\Delta$ long was positive (negative) if the station was to the east (west) of the $\Delta D=0$ longitude.

\section{Examples of Pi 2 Pulsations and the SUBSTORM CURRENT SYSTEM}

Before discussing the results of all 16 events statistically, two events are used to illustrate the analysis procedure. Pi 2 polarization parameters were determined both by plotting hodograms and by using spectral analysis techniques. Power spectra were computed for each field component at each station. The peak frequency in the range $5-25 \mathrm{mHz}(200-40$ s) was measured in the $H$ and $D$ components at each station. The dominant frequency at a majority of stations in each magnetic component was chosen as the overall dominant frequency. Polarization parameters of the Pi 2 pulsation, percent polarization, ellipticity, and azimuth were measured at the dominant frequency by using standard spectral analysis methods [see, e.g., Arthur et al., 1976]. The azimuth, or orientation of the major axis, is positive (negative) in the northeast (northwest) quadrant. The ellipticity is 0 when

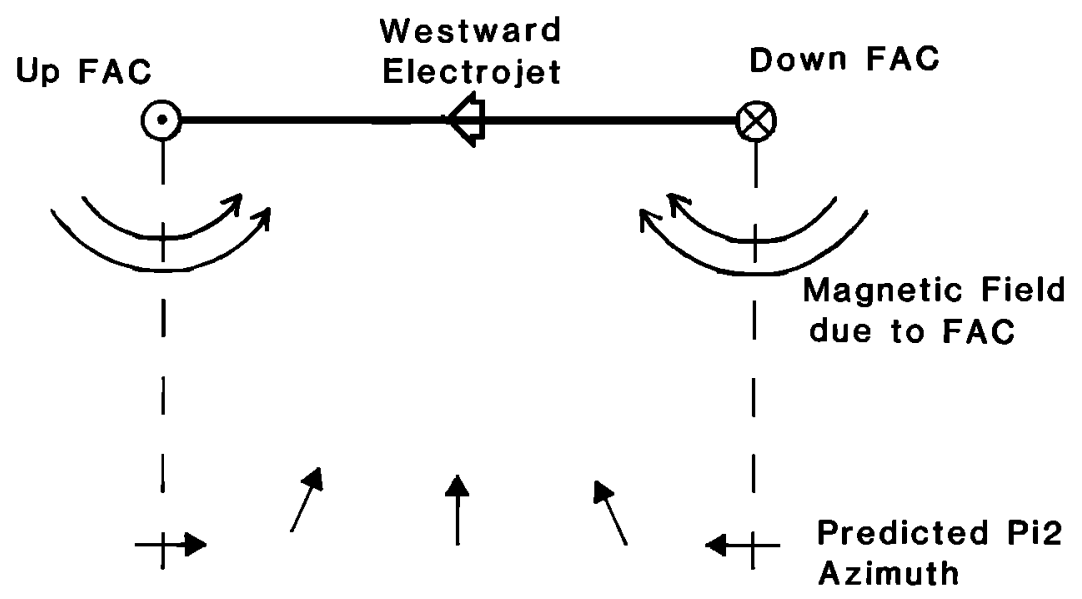

Fig. 3. A schematic view of the ionospheric and field-aligned portions of the substorm current wedge model shown in Figure 2. Also shown are the predicted Pi 2 polarization azimuths at mid-latitudes within the two extreme meridians of the current system if the Pi 2 is a result of the oscillation of such a current system. 

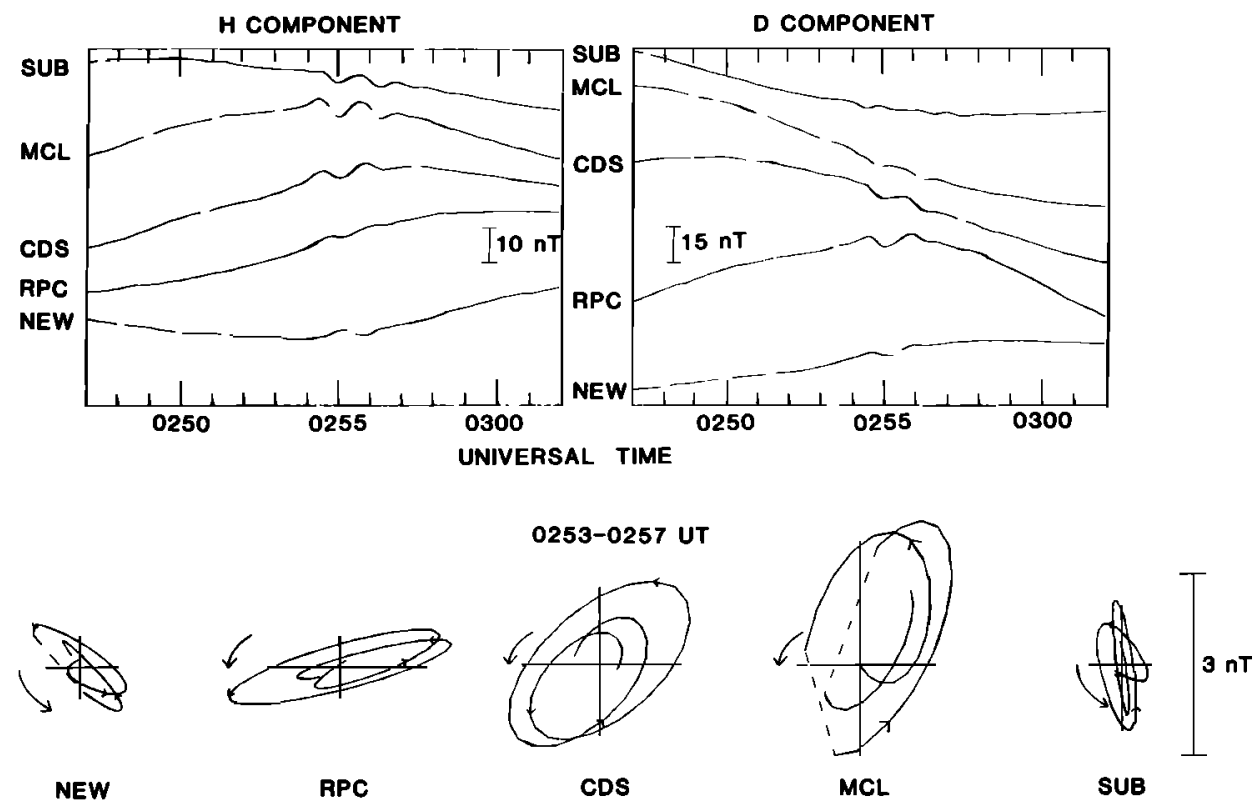

Fig. 4. A Pi 2 pulsation observed by the AFGL network in the interval 0247-0302 UT on March 14, 1978. In the upper panels the unfiltered $H$ and $D$ components show the Pi 2 pulsation and the magnetic bay. The lower panels show the hodograms measured during the interval 0253-0257 UT for each station. The dashed lines in some of the hodograms represent data gaps. The arrow at the side of each hodogram represents the sense of ellipticity. The hodograms at the four eastern stations compare favorably with the model Pi 2 azimuth pattern inside the wedge illustrated in Figure 3 . The polarization at station NEW is that expected for a station to the west of the wedge.

linear and 1 when circular. A positive (negative) ellipticity indicates a clockwise (anticlockwise) sense when looking down the magnetic field line in the northern hemisphere. Temporal variations of ellipticity and azimuth were examined by plotting hodograms. The two methods, hodograms and spectral wave analysis, produced similar results for the ellipticity and azimuth in all cases.

Figures 4 and 5 show unfiltered data for the time intervals 0247-0302 UT and 0555-0610 UT on March 14, 1978, each of which contains a Pi 2 pulsation. The lower panels show the hodograms for each event. During the earlier event (Figure
4), the hodogram at NEW, the station with the smallest wave amplitude, does not fit the predicted pattern inside the current wedge (Figure 3). However, it is consistent with the pattern predicted to the west of the western field-aligned current part of the wedge (not shown in Figure 3). At the other stations the polarization pattern is similar to the predicted one and points to a location between MCL and SUB. There is also some time variation of the azimuth at SUB, but the average azimuth fits the predicted pattern. For the later event, shown in Figure 5, the predicted pattern of the azimuth (Figure 3 ) is reproduced almost exactly.

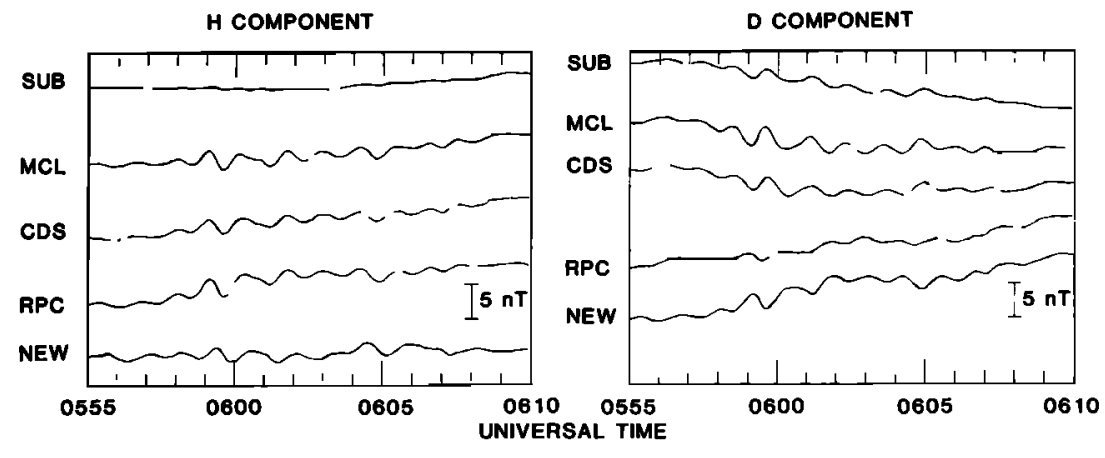

0559-0603 UT

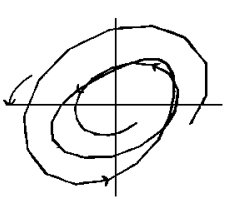

NEW

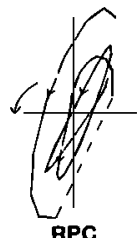

RPC

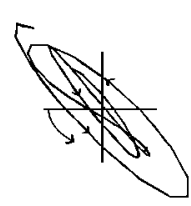

CDS

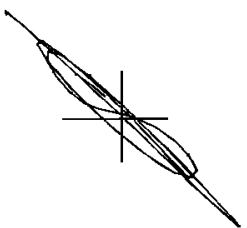

MCL

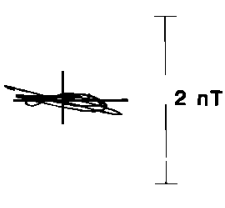

SUB

Fig 5. Same as Figure 4 for a Pi 2 pulsation in the interval 0555-0610 UT on March 14, 1978. When no arrow is present the ellipticity is essentially linear. The hodograms between 0559-0603 UT from all five stations compare favorably with the model shown in Figure 3. 
14 March 1978

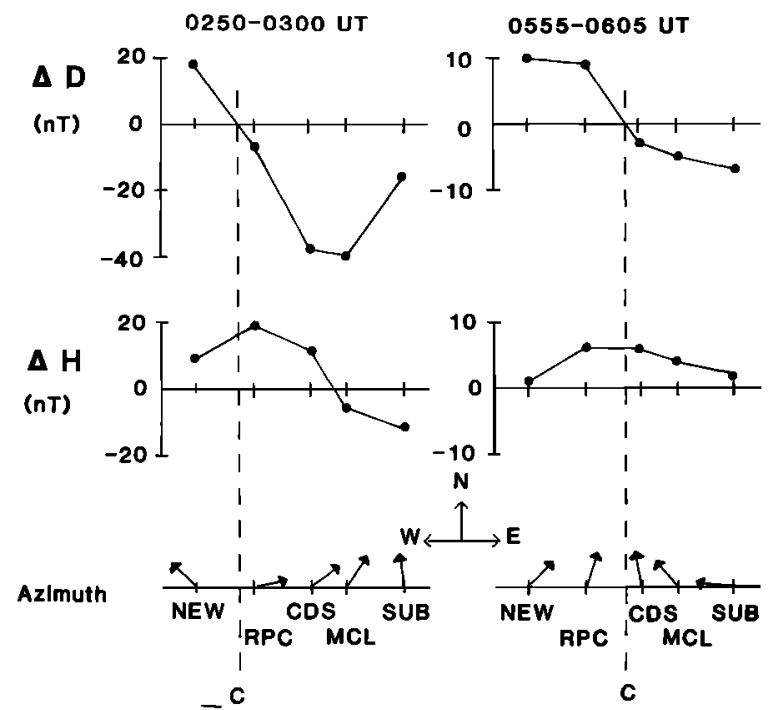

Fig. 6. The calculated values of $\Delta D$ (upper panels) and $\Delta H$ (middle panels) for the Pi 2 events in Figures 4 and 5 are shown. The longitudinal variation of $\Delta D$ and $\Delta H$ can be compared with the predicted mid-latitude signature in Figure 2. The location where $\Delta D$ $=0$, corresponding to the center of the wedge, is marked by a dashed line (C). The position of line $\mathrm{C}$ can be compared with the location where the major axis of the polarization ellipses (lower panels) points for the two events.

The bay structures (Figures 4 and 5, upper panels) show the schematic variation of the bays discussed in the previous section. The change in sign of the $D$ bay occurs between NEW and RPC for the 0247 UT event and between RPC and CDS for the 0555 UT event. Note also that, except for MCL and SUB, for the first event the $H$ bays are positive. The $D$ bay reaches the most negative value for $M C L$, which suggests that MCL and SUB are located at longitudes outside of the wedge.

The bay structure variation is further illustrated in Figure 6 by using the values of $\Delta H$ and $\Delta D$. For both events the upper panel shows the $\Delta D$ values for each station, and the middle panel shows the $\Delta H$ values. The position where $\Delta D=0$ is estimated, and it is marked by a dashed line indicating the wedge center (C). For the event at 0247 UT the line $\mathrm{C}$ passes between NEW and RPC, but the polarization pattern (bottom left panel), although similar to the predicted one, points between MCL and SUB, as mentioned earlier. This type of event, where the two methods of identifying the center of the wedge disagree, was found on five occasions. The size of the disagreement given in the example is the most extreme, but in all five cases the difference is more than a simple displacement to the wrong side of a station. The second event at 0555 UT shows that the center of the wedge identified by $\Delta D=0$ agrees with the center found by the polarization pattern. This type of event was found on 11 occasions.

\section{Polarization Variations Relative to the Substorm Current Wedge}

Having shown two examples of the polarization patterns of $\mathrm{Pi} 2$ pulsations, we now present the polarization parameters for the 16 events where the center of the current wedge was located between the NEW and SUB meridians. The azimuth and ellipticity were computed by using the spectral analysis method with 2.67 degrees of freedom. The conventions for azimuth and ellipticity were described in the previous section. Only values for which the percent polarization exceeded $95 \%$ were considered. If the modulus of the ellipticity $|\varepsilon|>0.80$, then the azimuth value was not considered, since the pulsation is nearly circularly polarized in such cases, and determining azimuth is imprecise [cf. Green and Hamilton, 1978, 1981].

The variation of azimuth for the 16 events where the center of the wedge is identified between NEW and SUB is shown in Figure 7. The azimuth at each station for each event is plotted in the substorm current wedge coordinate system where $\Delta$ long $=0$ is at the location of $\Delta D=0$. Only values that satisfied the selection criteria described in the above paragraph are plotted. The events where the two methods for defining the center of the substorm current wedge agree (dots) show the expected change of polarization across the wedge. For purposes of comparison the dashed line represents the Pi 2 azimuths expected from a substorm current wedge with a longitudinal extent of $90^{\circ}$ symmetric about the central meridian. The polarization azimuths for those events where the two methods disagree are indicated by crosses. The crosses corresponding to each event have been joined by straight lines. The remarkable feature is that these events also show a pattern similar to the dots but displaced relative to the wedge center. The displacement is not consistently to one side or the other of the dashed line,

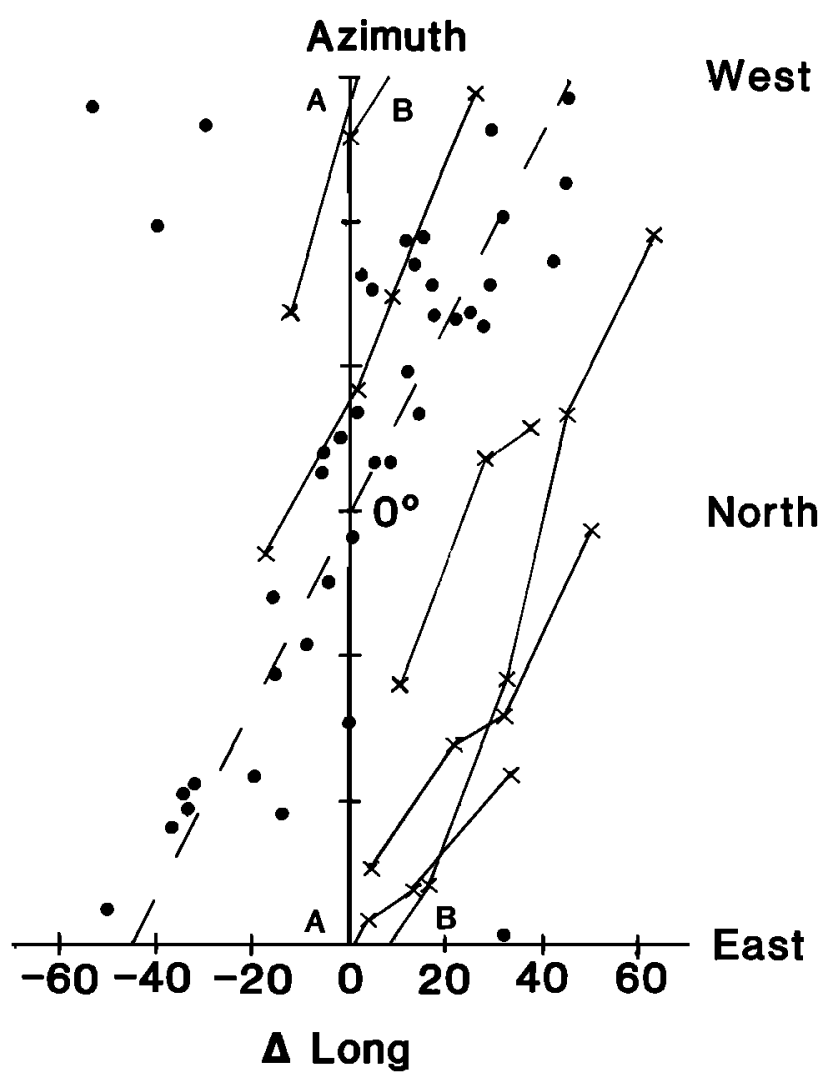

Fig. 7. Azimuth versus $\Delta$ long for the $16 \mathrm{Pi} 2$ events discussed in the study. Tick marks on the azimuth axis are every $30^{\circ}$. The dots represent the azimuth of the events where the two methods for locating the center of the current system agree. The crosses represent the azimuth for the events where the two methods disagree. The dashed line represents the variation expected for a model current system 6 hours wide and symmetric about the central meridian. The error in the measured azimuth value is $-10^{\circ}$. 


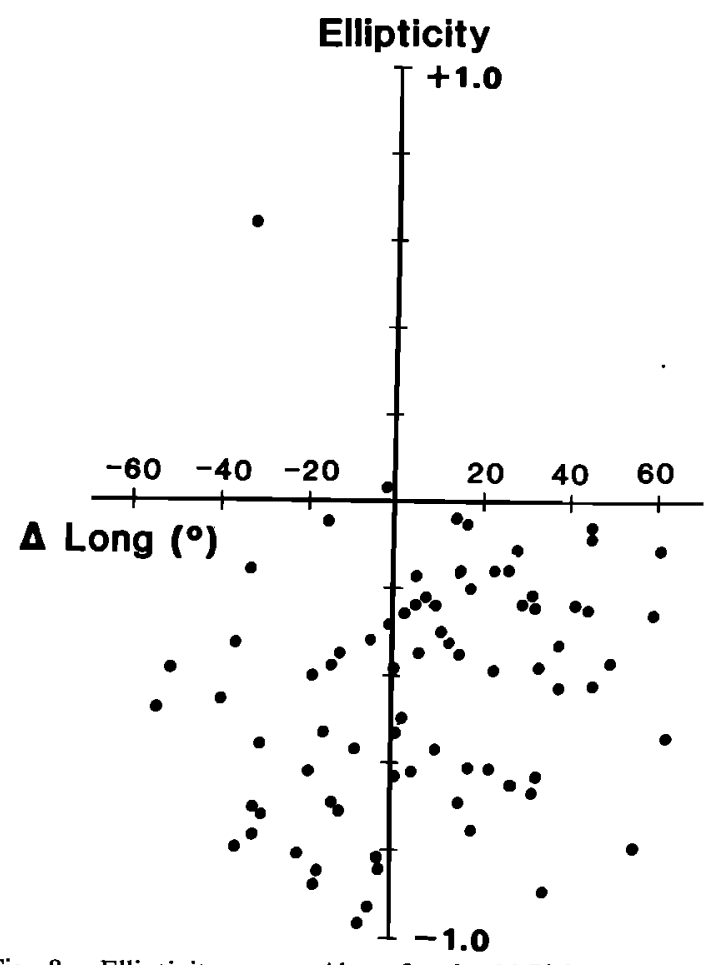

Fig. 8. Ellipticity versus $\Delta$ long for the $16 \mathrm{Pi} 2$ events discussed in the study. Positive (negative) ellipticity indicates a clockwise (anticlockwise) sense of rotation looking down the field line in the northern hemisphere. The center of the wedge is located at $\Delta$ long $=0$.

indicating the center of the Pi 2 current system can be either to the east or west of the substorm current wedge. It should be noted that, although we do not present the results, we found no systematic variation of azimuth with local time.

In the substorm current wedge model (Figure 3) the position where the azimuth of the magnetic perturbation is directed east-west locates the longitudes of the field-aligned current parts of the wedge. The difference in longitude between the field-aligned current portions can be regarded as the effective width of the wedge. Since it is unusual for the entire width of the wedge to be located within the two longitudinal extremes of the network, the wedge width was estimated from the slope of the lines connecting the dots or crosses for each individual event. This slope varies from $\sim 1^{\circ}$ to $\sim 2.5^{\circ}$ azimuth/degree longitude, with an average of $\sim 2^{\circ}$ azimuth/degree longitude. This implies a variation in the wedge width of between $\sim 4$ and $\sim 12$ hours and an average of $\sim 6$ hours. These results are in reasonable agreement with the observations of Clauer and McPherron [1974b], who found the extent of the current system to vary between 2.5 and 10 hours, with an average of just over 4 hours.

The ellipticity variation with respect to $\Delta$ long is shown in Figure 8, where all but two values are negative. The predominance of anticlockwise values agrees with other results for stations at these latitudes [e.g., Fukinishi, 1975; Mier-Jedrzejowicz and Southwood, 1979; Lester and Orr, 1981]. Apart from this, no ordering appears in the sense of polarization with respect to $\Delta$ long. Also, we found no systematic local time variation of ellipticity.

\section{Phase Differences of Pi 2 Pulsations Between} STATIONS

The longitudinal variation of pulsation phase has been discussed in the past in terms of the $m$ number [e.g., Green,
1976; Mier-Jedrzejowicz and Southwood, 1979]. The $m$ number is obtained by dividing the phase difference between a pair of stations by the station longitudinal separation. It has then been interpreted in terms of an apparent azimuthal wave number. However, since the azimuthal propagation of the Pi 2 signal is not clear, we shall not interpret the $m$ number in this way but simply consider it as the phase difference per degree of longitude.

The phase differences between all possible pairs of stations were calculated by using a cross-spectral analysis technique [Hughes et al., 1978]. Only the phase differences between adjacent station pairs are used here. The $95 \%$ confidence limits in the phase difference were calculated by using the coherency value and the number of degrees of freedom associated with the smoothing parameter used in the spectral computation [Green, 1976]. We calculated the $m$ number from the calculated phase difference and an error in $m, \Delta m$, from the confidence limits in the phase difference.

Figure 9 shows the $m$ values for the 11 events where the two methods of identifying the center of the substorm current wedge agree. The error bar, representing $\Delta m$, is plotted if $\Delta m>|m|$, i.e., there is ambiguity in the sign of $m$. We included only those points for which $\Delta m<1.5$. This value was chosen as a compromise between having sufficient number of points and sufficient resolution in $m$. The maximum error allowed, $\Delta m=1.5$, is also shown. The $H$ component (Figure $9 a$ ) shows a weak tendency for $|m|$ to decrease from west to east across the wedge. The $D$ component (Figure $9 b$ ) shows a similar tendency for the values to the east of the center to decrease with increasing $\Delta$ long. The larger $|m|$ values are west of center of the wedge for the $H$ component and close to the center for the $D$ component.

The $m$ values are predominantly negative, irrespective of the location of the center of the substorm current wedge and local time (results not presented). This agrees with the work of Mier-Jedrzejowicz and Southwood [1979], Warner [1979], and Green and Stuart [1979]. Mier-Jedrzejowicz and Southwood did not use individual events, but power levels in the Pi 2 frequency band averaged over 1 hour time intervals. Warner, however, studied individual Pi 2 events observed with the same network as Mier-Jedrzejowicz and Southwood. Baranskiy et al. [1980] reported that there was a slight tendency for $m$ to change sign near midnight. They have shown the $\boldsymbol{H}$ component of the western station leading before midnight and the $D$ component of the western station leading after midnight. Neither reversal is seen in our results. Also, there is no sign of the reversal reported by Herron [1966], who used data from a similar longitude sector and latitude as the AFGL network.

\section{Discussion}

For each of the 16 events studied in detail the azimuths of the major axis of the polarization ellipses agree with the pattern predicted by a model three-dimensional current system (Figure 2). The pattern was better organized by the substorm current wedge and not by local time. Measurements made by Green and Stuart [1979; personal communication, 1980] and Baranskiy et al. [1980] support our results. Two examples of the azimuth of the major axis of the polarization ellipse [see Figure 5, Baranskiy et al., 1980] illustrate the pattern predicted by a current wedge model. Furthermore, using many events, Baranskiy et al. did not find a systematic variation with local time (see their Figure 


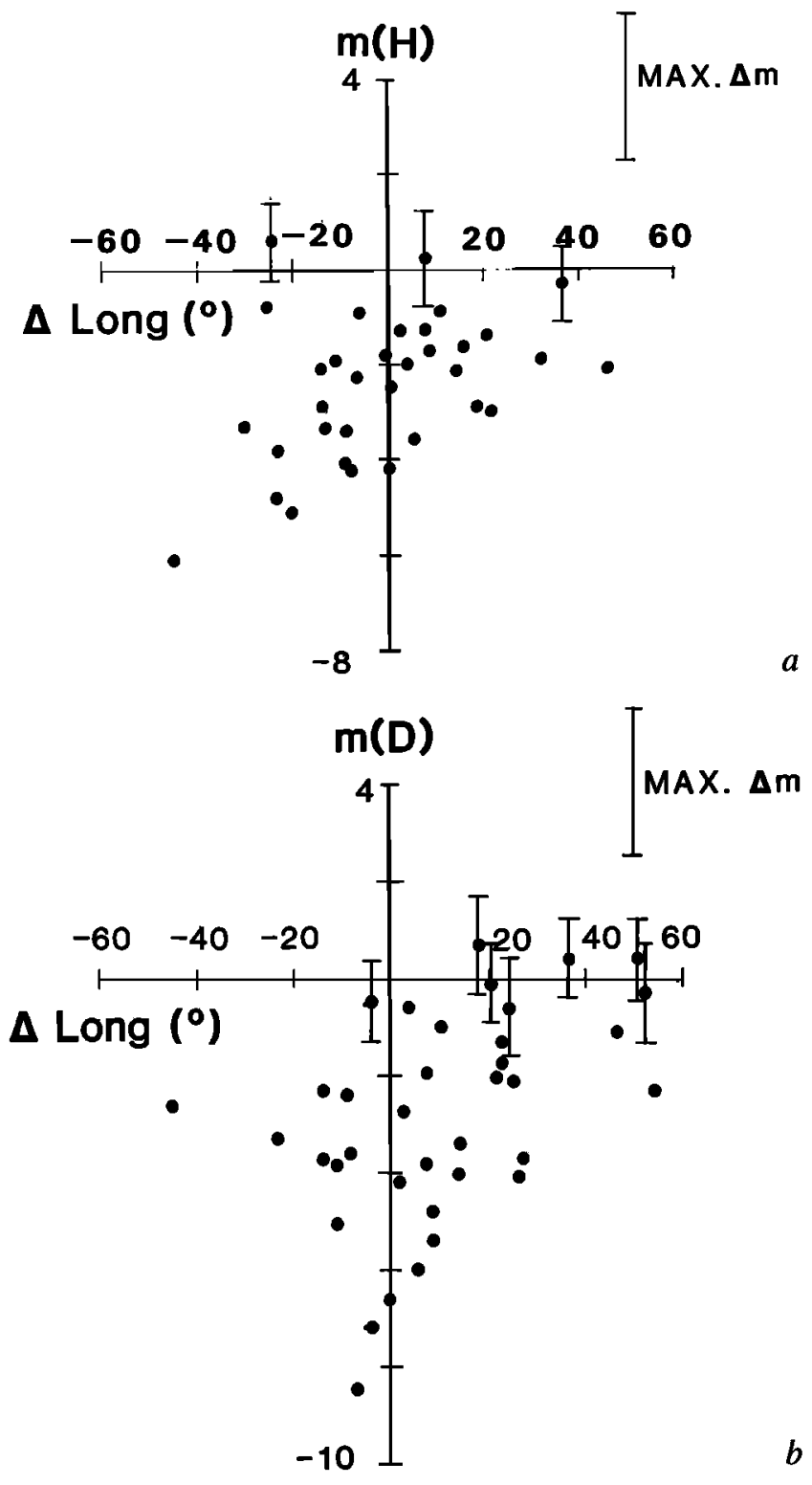

Fig. 9. The phase difference per degree of longitude, $m$ value, between pairs of stations plotted against the $\Delta$ long of the midpoints of the two stations for $H(9 a)$ and $D(9 b) ; m$ is calculated by using only adjacent station pairs for the 11 events where the two methods of locating the center of the current system agree. Positive (negative) $m$ indicates the western (eastern) station leads in phase. Error bars are marked if $\Delta m>|m|$ and the maximum error in any value plotted is 1.5 .

$6 c)$. However, there have been some studies which suggest a local time organization of the Pi 2 polarization pattern [e.g., Bjornsson et al., 1971; Fukinishi, 1975; Stuart and Baranskiy, 1982]. Bjornsson et al. [1971], using averages of Pi 2 azimuths determined in 1 hour local time bins at stations between $40^{\circ}$ and $50^{\circ}$ magnetic latitude, found a pattern similar to that in Figure 3, but pointing to the $2200 \mathrm{LT}$ meridian. These correlations between Pi 2 azimuths and local time would be expected if the centers of the current systems associated with the pulsations used in a study had a small local time spread. We are unable to test this explanation because the studies showing a local time organization of the $\mathrm{Pi} 2$ azimuths do not indicate the location of the wedge centers. In this paper the centers of the current systems were found to range from 1940 to $0100 \mathrm{LT}$, a range that makes it possible to distinguish between a local time or current wedge organization of the data.

The occasional disagreement between the center of the wedge identified by the two methods implies that if the Pi 2 pulsation is associated with a three-dimensional current system then that current system is not always colocated with the substorm current system. A study by Pashin et al. [1982] appears to support this result, as they also conclude that the wave and substorm current systems are offset. However, since they were using data from a small region at high latitudes, the exact relationship between their results and ours requires further study. Alternatively, the Pi 2 could be linked to the substorm current system only at onset (J. C. Samson, personal communication, 1982). After onset there often is a westward expansion of the current system, which will affect the bay at mid-latitudes. In this case the center identified by $\Delta D=0$ would be to the west of that identified by the $\mathrm{Pi} 2$ polarization pattern. This happens in four out of five of the cases where the two methods disagreed.

As previously mentioned, we believe the wedge current system discussed in this paper is an oversimplification of the real current systems [Baumjohann et al., 1981]. There are several situations that could account for discrepancies between observations and model. First, there are likely to be current systems located outside the current wedge. Second, preexisting currents in the vicinity of the substorm current wedge can confuse the bay pattern. Third, the currents may be distributed in both latitude and longitude. Nevertheless, we have demonstrated that the simple wedge model often orders the data well.

In this paper we are not attempting to completely describe the currents that are an integral part of the Pi 2 wave. Since $\mathrm{Pi} 2$ polarizations are generally elliptical, the current patterns must move periodically. What we have found is that the largest magnetic perturbations, those corresponding to the major axes of the polarization ellipses, do fit a current topologically similar to the substorm current system. The magnetic signature seen at one ground station describes the integrated effect of currents flowing in a substantial region of the ionosphere. If the ionosphere conductivity is uniform, the ground signature results from Hall currents alone [Hughes and Southwood, 1976]. However, at night the conductivity is far from uniform; the auroral zone can have a substantially higher conductivity than the surrounding ionosphere. In this case the ground signature can only be determined by a full Biot-Savart integration of the ionospheric and field-aligned currents. The inverse problem is inherently nonunique. A full description of the currents associated with a Pi 2 pulsation must await further study.

The predominance of negative $m$ values in both $H$ and $D$ components found in section 6 agrees with Mier-Jedrzejowicz and Southwood [1979] and Warner [1979] but not Herron [1966]. Previously, $m$ values have been interpreted as plane-wave wave numbers, which in this case implies westward phase propagation across the entire nightside. We consider it dangerous to interpret $m$ values in this way. To do so implies a Pi 2 wave source on the morningside of the magnetosphere, and we have seen no substorm model that can incorporate this interpretation, since substorms are a nightside phenomenon with a peak occurrence between 22 LT and local midnight.

Finally, the weak tendency of $|m|$ to decrease to the east of the center of the wedge is consistent with the eastern field- 
aligned currents being located over a wider longitudinal range than the more intense upward field-aligned currents at the western part of the current system. This result is suggested by the tendency for localized structures to be associated with higher $m$ numbers. Such a current system has been implied from ground-based magnetometer measurements and STARE electric field measurements in Scandinavia [Baumjohann et al., 1981]. Westward motion of such a current system relative to the ground observations would mean that the phase change would be larger at the western part of the array, and the eastern stations would lead in phase. Such motion of the substorm current system does seem to occur [e.g., Wiens and Rostoker, 1975].

\section{Summary and Conclusions}

Results from a study of Pi 2 pulsations observed on an east-west chain of magnetometers have been presented. We have concentrated on the relationship between magnetic bays and $\mathrm{Pi} 2$ polarization characteristics. We also calculated the phase differences between adjacent station pairs. We can summarize the results as follows:

1. The magnetic bays observed at mid-latitude ground stations can be used to locate the center of the substorm current wedge.

2. A predicted Pi 2 polarization pattern based on a current wedge model is observed in each of 16 events.

3. Two methods for locating the center of the substorm current wedge agreed in 11 out of 16 cases.

4. Using the Pi 2 azimuths, the effective width of the $\mathrm{Pi} 2$ current system was estimated to have an average value of 6 hours for the 16 events.

5. There is no systematic variation in ellipticity with either local time or the substorm current system.

6. Wave phase differences between stations, or $m$, are predominantly negative in both $H$ and $D$ components.

7. Wave phase difference between stations tends to decrease to the east of the center of the substorm current wedge, consistent with western upward field-aligned currents being more localized than the eastern downward currents.

The simple three-dimensional substorm current wedge model orders Pi 2 polarization data well. The relationship between the Pi 2 polarization and the substorm bay shows that the two current systems are not always colocated. This is important both in terms of the generation mechanism of the Pi 2 pulsation and also in gross substorm dynamics. Further study of this property will be carried out, and it is hoped that the results will provide more information on the processes involved in the generation of $\mathrm{Pi} 2$ pulsations and their relationship with substorm onset.

Acknowledgments. This work was supported under U.S. Air Force contract F19628-81-K-003. We appreciate the efforts of D. J. Knecht for operating the AFGL network and initial data processing. We would like to acknowledge helpful discussions with J. C. Samson.

The Editor thanks W. F. Stuart and K. H. Glassmeier for their assistance in evaluating this paper.

\section{REFERENCES}

Arthur, C. W., R. L. McPherron, and J. D. Means, A comparative study of three techniques for using the spectral matrix in wave analysis, Radio Sci., II, 833, 1976.

Baranskiy, L. N., V. A. Troitskaya, I. V. Sterlikova, M. B.
Gokhberg, N. A. Ivanov, I. P. Khartchenko, J. W. Munch, and $\mathrm{K}$. Wilhelm, The analysis of simultaneous observations of nighttime $\mathrm{Pi}$ pulsations on an east-west profile, J. Geophys., 48, 1 , 1980.

Baumjohann, W., R. J. Pellinen, H. J. Opgenoorth, and E. Nielsen, Joint two-dimensional observations of ground magnetic and ionospheric electric fields associated with auroral zone currents: Current systems associated with local auroral break-ups, Planet. Space Sci., 29, 431, 1981.

Bjornsson, A., O. Hillebrand, and H. Voelker, First observational results of geomagnetic $\mathrm{Pi} 2$ and Pc5 pulsations on a north-south profile through Europe, Z. Geophys., 37, 1031, 1971.

Bostrom, R., Magnetosphere-ionosphere coupling, in Critical Problems of Magnetospheric Physics, edited by E. R. Dyer, National Academy of Sciences, Washington, D.C., 1972.

Clauer, C. R., and R. L. McPherron, Mapping the local timeuniversal time development of magnetospheric substorms using mid-latitude magnetic observatories, J. Geophys. Res., 79, 2811, $1974 a$.

Clauer, C. R., and R. L. McPherron, Variability of mid-latitude magnetic parameters used to characterize magnetospheric substorms, J. Geophys. Res., 79, 2898, $1974 b$.

Fukunishi, H., Polarization changes of geomagnetic Pi2 pulsations associated with the plasmapause, J. Geophys. Res., 80, 98, 1975.

Green, C. A., The longitudinal phase variation of midlatitude Pc 3-4 micropulsations, Planet. Space Sci., 24, 79, 1976.

Green, C. A., and R. A. Hamilton, Polarization characteristics and phase differences of $\mathrm{Pi} 2$ pulsations at conjugate stations, $J$. Atmos. Terr. Phys., 40, 1223, 1978.

Green, C. A., and R. A. Hamilton, An ionospheric effect on the conjugate relationship of $\mathrm{Pi} 2$ magnetic pulsations, J. Atmos. Terr. Phys., 43, 1133, 1981.

Green, C. A., and W. F. Stuart, Apparent phase velocities of Pi 2's, paper presented at IAGA General Assembly, Canberra, Australia, 1979.

Herron, T. J., Phase characteristics of geomagnetic micropulsations, J. Geophys. Res., 7I, 871, 1966.

Hughes, W. J., and D. J. Southwood, The screening of micropulsation signals by the atmosphere and ionosphere, J. Geophys. Res., $81,3234,1976$.

Hughes, W. J., R. L. McPherron, and J. N. Barfield, Geomagnetic pulsations observed simultaneously on three geostationary satellites, J. Geophys. Res., 83, 1109, 1978.

Knecht, D. J., Pulsations from small multiple-onset substorms observed by a long east-west chain of stations (abstract), EOS Trans. AGU, 62, 1014, 1981.

Knecht, D. J., R. O. Hutchinson, and C. W. Tsacoyeanes, An introduction to the AFGL magnetometer network, report, Plasma, Particles, and Fields Br., Space Phys. Div., Air Force Geophys. Lab., Hanscom A.F.B., Mass., 1979.

Kuwashima, M., and T. Saito, Spectral characteristics of magnetic $\mathrm{Pi} 2$ pulsations in the auroral region and lower latitudes, $J$. Geophys. Res., 86, 4686, 1981.

Lester, M., and D. Orr, The spatio-temporal characteristics of Pi2's, J. Atmos. Terr. Phys., 43, 947, 1981.

McPherron, R. L., C. T. Russell, and M. P. Aubry, Satellite studies of magnetospheric substorms on August 15, 1968, 9, Phenomenological model for substorms, J. Geophys. Res., 78, 3131, 1973.

Mier-Jedrzejowicz, W. A. C., and D. J. Southwood, The east-west structure of midlatitude geomagnetic pulsations in the $8-25 \mathrm{mHz}$ band, Planet. Space Sci., 27, 617, 1979.

Pashin, A. B., K. H. Glassmeier, W. Baumjohann, O. M. Raspopov, A. G. Yahnin, H. J. Opgenoorth, and R. J. Pellinen, Pi 2 magnetic pulsations, auroral break-ups and the substorm current wedge: A case study, J. Geophys., 51, 223, 1982.

Rostoker, G., and J. V. Olson, Pi 2 micropulsations as indicators of substorm onsets and intensifications, in Auroral Processes, edited by C. T. Russell, Japan Scientific Societies Press, Tokyo, 1979.

Rostoker, G., and J. C. Samson, Polarization characteristics of Pi 2 pulsations and implications for their source mechanisms: Location of source regions with respect to the auroral electrojets, Planet. Space Sci., 29, 225, 1981.

Rostoker, G., S.-I. Akasofu, J. Foster, R. A. Greenwald, Y. Kamide, K. Kawasaki, A. T. Y. Lui, R. L. McPherron, and C. T. Russell, Magnetospheric substorms-Definition and signatures, J. Geophys. Res., 85, 1663, 1980.

Saito, T., Oscillation of geomagnetic field with the progress of pt- 
type pulsations, Sci. Rep. Tohoku Univ., 5, Geophys., 13, 53, 1961.

Saito, T., K. Yumoto, and Y. Koyama, Magnetic pulsation Pi2 as a sensitive indicator of magnetospheric substorm, Planet. Space Sci., 24, 1025, 1976.

Samson, J. C., Pi 2 pulsations: High latitude results, Planet. Space Sci., 30, 1239, 1982.

Samson, J. C., and B. G. Harrold, Maps of the polarization of high latitude Pi 2's, J. Geophys. Res., 88, 5736, 1983.

Samson, J. C., and G. Rostoker, Polarization characteristics of Pi 2 pulsations and implications for their source mechanisms, 2, Influence of the westward travelling surge, Planet. Space Sci., 3I, $435,1983$.

Singer, H. J., W. J. Hughes, P. F. Fougere, and D. J. Knecht, The localizaiton of Pi 2 pulsations: Ground-satellite observations, $J$. Geophys. Res., 88, 7029-7036, 1983.

Stuart, W. F., and L. N. Baranskiy, Simultaneous observations of Pi 2 pulsations on N-S meridians in the U.K. and Scandinavia, submitted to J. Atmos. Terr. Phys., 1982.

Sutcliffe, P. R., The longitudinal range of $\mathrm{Pi} 2$ propagation at low latitudes, Planet. Space Sci., 28, 9, 1980.

Warner, M. R., A study of the spatial characteristics of long period geomagnetic micropulsations at mid and high latitudes, D. Phil. thesis, Univ. York, U.K., 1979.

Wiens, R. G., and G. Rostoker, Characteristics of the development of the westward electrojet during the expansive phase of magnetospheric substorms, J. Geophys. Res., 80, 2109, 1975.

W. J. Hughes and M. Lester, Department of Astronomy, Boston University, Boston, MA 02215.

H. J. Singer, Air Force Geophysics Laboratory, Plasma, Particles and Fields Branch, Hanscom A.F.B., MA 01731.

(Received March 23, 1983;

revised June 9, 1983;

accepted June 10, 1983.) 